\title{
A note on the summation of an infinite series involving a hypergeometric function
}

\section{A.G. Williamson}

\begin{abstract}
The sum of an infinite series involving the ${ }_{4}{ }_{3}$ function is found by considering an infinite series whose terms involve the product of two Bessel functions of the first kind. Furthermore the infinite series involving the ${ }_{4}{ }_{3}$ function can be utilized to find an approximation to the ${ }_{4} F_{3}$ function of unit argument, for particular values of the parameters.
\end{abstract}

The mathematical properties of the generalized hypergeometric function are now well established, due largely to the efforts of Bailey, Watson, slater, and many others. There are available a number of books on the subject [1], [6] which contain extensive bibliographies together with an outline of the history of the investigations. A large portion of the published work concerning these functions has in fact dealt with the Gauss hypergeometric function and, as a consequence, many interesting results have been documented. Such results include the evaluation of the function for special values of parameters and argument, asymptotic expansions and so on. The situation, in this regard, for other hypergeometric functions is not as satisfactory. The exception is Kummer's function which like the Gauss function has been well researched.

Generalised hypergeometric functions are frequently encountered in the solution of physical problems and the ease of numerical evaluation of these

Received 18 December 1973. 
functions often determines the practicability of the solution method. As such, the establishment of evaluation techniques for these functions is of considerable interest to engineers, physicists, and applied mathematicians. Some attention has recently been focused on the development of approximation formulae [2] and recently Luke [3] developed simple formulae for cases with negative argument. Such approximations, while extremely useful, are often difficult to apply. Furthermore these approximations, while including many cases of interest, often impose restrictions which exclude many of the more frequently encountered forms. In particular the evaluation of the generalised hypergeometric function for unit argument (in the cases where the series converges) is often required and approximation formulae are not well suited, if indeed applicable, to this requirement. With this in mind simple methods of evaluating these functions for special cases of parameters and argument are most valuable.

In particular this paper investigates the summation of an infinite series involving the ${ }_{4} F_{3}$ function. Furthermore the result can be used to find an approximation, to which error bounds can be established, for the $4_{3}{ }_{3}$ function of unit argument, for special values of the parameters.

We begin by investigating the series

$$
S=\sum_{m=1}^{\infty} \frac{J_{2 \alpha}(m c) J_{2 \beta}(m c)}{m(m c)^{2 \gamma}},
$$

where $2 \alpha+2 \beta>1+2 \gamma$. (This form of series has recently been encountered by the author in connection with the study of the electromagnetic behaviour of cylindrical antennas in rectangular waveguides.) Utilizing Poisson's summation formula, $S$ may be rewritten as

$$
s=s_{a}+s_{b}
$$

where

$$
S_{a}=\int_{0}^{\infty} \frac{J_{2 \alpha}(c z) J_{2 \beta}(c z)}{z(c z)^{2 \gamma}} d z
$$

and

$$
S_{b}=2 \sum_{m=1}^{\infty} \frac{J_{2 \alpha}(c z) J_{2 \beta}(c z)}{z(c z)^{2 \gamma}} \cos (2 m \pi z) d z
$$


The term $S_{a}$ is in fact a Weber-Schafheitlin discontinuous integral which can be written in terms of Gamma functions [4, p. 99], namely

$$
S_{a}=\frac{1}{2^{1+2 \gamma}} \cdot \frac{\Gamma(\alpha+\beta-\gamma)}{\Gamma(1+\alpha+\beta+\gamma) \Gamma(1+\alpha+\gamma-\beta) \Gamma(1+\beta+\gamma-\alpha)} .
$$

To evaluate $S_{b}$, the result that

$$
J_{\nu}(z) J_{\mu}(z)=2 / \pi \int_{0}^{\pi / 2} J_{\mu+\nu}(2 z \cos \theta) \cos (\nu-\mu) \theta d \theta
$$

for $\mu+\nu>-1$ is used, such that

$$
S_{b}=4 / \pi \sum_{m=1}^{\infty} \int_{0}^{\infty} \frac{\cos (2 m \pi z)}{z(c z)^{2 \gamma}} d z \cdot \int_{0}^{\pi / 2} J_{2 \alpha+2 \beta}(2 c z \cos \theta) \cos (2 \alpha-2 \beta) \theta d \theta .
$$

On changing the order of integration, which presents no difficulty, a Fourier cosine transform needs to be evaluated. This transform, which is tabulated [5, p. 63], can be evaluated in terms of the Gauss hypergeometric function. On expanding the Gauss function termwise, performing the remaining integral, and using the definition of the ${ }_{4}{ }_{3}$ function, $S_{b}$ can be shown to be

$$
\begin{aligned}
& \text { (3) } S_{b}=2^{1-2 \alpha-2 \beta} \cos [(\alpha+\beta-\gamma) \pi] \cdot \frac{\Gamma(2 \alpha+2 \beta-2 \gamma)}{\Gamma(1+2 \alpha) \Gamma(1+2 \beta)} \cdot \sum_{m=1}^{\infty}\left(\frac{c}{2 m \pi}\right)^{2 \alpha+2 \beta-2 \gamma} \\
& { }_{4} F_{3}\left(\alpha+\beta-\gamma, \alpha+\beta-\gamma+\frac{1}{2}, \frac{2}{2}+\alpha+\beta, 1+\alpha+\beta ; 1+2 \alpha+2 \beta, 1+2 \alpha, 1+2 \beta ;(c / m \pi)^{2}\right)
\end{aligned}
$$

where $2 \alpha+2 \beta>1+2 \gamma>-\frac{3}{2}$.

It is interesting to note that (3) may be rearranged to be

$$
\text { (4) } \begin{aligned}
& S_{b}=\frac{\cos [(\alpha+\beta-\gamma) \pi]}{\pi} \cdot\left(\frac{c}{\pi}\right)^{2 \alpha+2 \beta-2 \gamma} \cdot \sum_{n=0}^{\infty} \frac{\Gamma(\alpha+\beta-\gamma+n) \Gamma\left(\alpha+\beta-\gamma+\frac{2}{2}+n\right) \Gamma(1+\alpha+\beta+n)}{\Gamma(1+2 \alpha+2 \beta+n) \Gamma(1+2 \alpha+n) \Gamma(1+2 \beta+n)} \\
& \cdot \frac{\Gamma\left(\frac{1}{2}+\alpha+\beta+n\right)}{n !}\left(\frac{c}{\pi}\right)^{2 n} \zeta(2 \alpha+2 \beta-2 \gamma+2 n)
\end{aligned}
$$

where $\zeta(x)$ is a Riemann Zeta function. Equation (4) is an extremely efficient and convenient way of numerically calculating $S_{b}$ for cases where $\left(\frac{c}{\pi}\right)$ is small. Note that for $\left(\frac{c}{\pi}\right)$ small direct calculation of $S$ by (I) is impractical.

Now reconsider (1) for the special case 


$$
2 \beta=\frac{1}{2}, c=\pi,
$$

whereupon every term of (1) is zero since

$$
J_{\frac{1}{2}}(m \pi)=0, m=1,2,3, \ldots ;
$$

hence

$$
S_{b}=-S_{a}
$$

With appropriate substitutions the result

(5) $\sum_{m=1}^{\infty}\left(\frac{1}{m}\right)^{2 a} 4^{F}\left(a, \frac{1}{2}+a, c, \frac{1}{2}+c ; 2 c-\frac{1}{2}, 2 c, \frac{3}{2} ; \frac{1}{m^{2}}\right)=\frac{2^{4 c-3} \Gamma\left(2 c-\frac{1}{2}\right)}{\sqrt{\pi}(2 a-1) \Gamma(4 c-2 a-1)}$

$$
=f(a, c)
$$

may be deduced, the restrictions on $a$ and $c$ being

$$
2 c+\frac{1}{2}>2 a>1 \text {. }
$$

Inspection of (5) reveals that for large $a$ the terms of the infinite series decay very rapidly. This property can be used to find an approximation to the $4_{3}$ function of unit argument. Furthermore an error bound to this approximation may be established by noting, for the case where all the $\rho^{\prime} s$ and $\sigma^{\prime} s$ are positive, that

$$
{ }_{4} F_{3}((\rho) ;(\sigma) ; x)>{ }_{4} F_{3}((\rho) ;(\sigma) ; y)
$$

if $x>y \geq 0$. Hence for all $a>\frac{1}{2}, c>a-\frac{3}{4}$,

(6) $f(a, c)-\varepsilon(a, c)<{ }_{4} F_{3}\left(a, \frac{3}{2}+a, c, \frac{1}{2}+c ; 2 c-\frac{1}{2}, 2 c, \frac{3}{2} ; 1\right) \leq f(a, c)$

where $\varepsilon(a, c)$ is given by

$$
\varepsilon(a, c)=\frac{f(a, c)+3}{4} \cdot(\zeta(2 a)-1) .
$$

Note that $\varepsilon(a, c) \ll f(a, c)$ for large $a$.

It has been shown that the sum of an infinite series involving the $4_{3} F_{3}$ function can be deduced by investigating the sum of an infinite series involving the product of two Bessel functions of the first kind. Furthermore an approximation, together with an error bound, may be deduced for the $4_{3} F_{3}$ function of unit argument for special values of the parameters.

It is worth pointing out that other special cases of (1) could also 
be cast into forms similar to (5) such that approximations to the ${ }_{4} F_{3}$ of unit argument, but of other parameters, could be obtained. Indeed it is possible to build up a family of such approximations using (1). Details of the work will be published shortly.

It appears to the author that the method outlined in this paper could profitably be applied to other series similar to (1) with a view to obtaining approximation formulae for other hypergeometric functions. It is the author's experience that approximations similar to (6) find considerable application in the applied sciences.

\section{References}

[1] Arthur Erdélyi, Wi thelm Magnus, Fritz Oberhettinger, Francesco G. Tricomi (edited by), Higher transcendental functions, I. Based, in part, on notes left by Harry Bateman. (McGraw-Hill, New York, Toronto, London, 1953.)

[2] U.J. Knottnerus, Approximation formulae for generalized hypergeometric functions for large values of the parcmeters (Wolters, Groningen, 1960).

[3] Yudell L. Luke, "Inequalities for generalized hypergeometric functions", Aerospace Research Labs. Report 70-0041, March 1970.

[4] Wilhelm Magnus, Fritz Oberhettinger, Raj Pal Soni, Formulas and theorems for the special functions of mathematical physics, 3 d d enlarged edition (Die Grundlehren der mathematischen Wissenschaften, Band 52. Springer-Verlag, Berlin, Heidelberg, New York, 1966).

[5] Fritz Oberhettinger, Tabelzen zur Fourier Transformation (SpringerVerlag, Berlin, Göttingen, Heidelberg, 1957).

[6] Lucy Joan Slater, Generalized hypergeometric functions (Cambridge University Press, Cambridge, 1966).

Department of Electrical Engineering, University of Auckland, Auckland, New Zealand. 